REFERENCES AND/OR ACKNOWLEDGEMENTS

No conflict of interest.

\section{CPS-153 INTENSIFICATION IN BIOLOGICAL TREATMENT IN ULCERATIVE COLITIS}

A Pintado*, R Asensi Diez, A Linares, I Muñoz Castillo. Hospital Regional Universitario Malaga, Farmacia Hospitalaria, Malaga, Spain

\subsection{6/ejhpharm-2020-eahpconf.254}

Background and importance Biological drugs have improved the therapeutic possibilities for ulcerative colitis (UC), showing good clinical efficacy. However, a considerable percentage of patient do not initially respond to treatment or lose the response achieved over time. To resolve treatment failure, several strategies have been used, including intensification of treatment.

Aim and objectives To analyse the use of biological drugs in patients with UC and the strategies used in the intensification of these treatments in clinical practice.

Material and methods This was a retrospective observational study. Inclusion criteria were patients with UC who received biological treatment during the study period (in 2018). Variables collected were sex, age, number of years diagnosed, intestinal inflammation marker (calprotectin (CF)) before and after treatment, biological drug received during the study period, use of intensification and strategy used (dose increase or dosage interval shortening and determination of drug levels). Loss of response was defined as therapeutic levels not achieved in the case of infliximab (IFX) and adalimumab (ADA). Data were obtained from the outpatient dispensing programme (ATHOS) and the electronic medical records (Diraya).

Results During the study period, 48 patients were included: $61.54 \%$ women, median age 41 years (range 19-64) and median number of years diagnosed 7 years (range 1-29). Median CF before starting treatment was 513.95 (range 1284257) and after biological treatment it was 97 (range 83963).

The prescribed biological drugs were IFX in $53.06 \%$ of patients $(n=26)$, ADA in $22.44 \%(n=11)$, vedolizumab (VDZ) in $14.29 \%(\mathrm{n}=7)$, tofacitinib in $4.08 \%(\mathrm{n}=2)$ and ustekinu$\mathrm{mab}$ in $4.08 \%(\mathrm{n}=2)$. Treatment was intensified in $46.93 \%$ of patients (IFX $n=16$; ADA $n=1$; VDZ $n=6$ ) due to loss of response. In all patients the intensification strategy was to shorten the dosing interval except in two cases in whom the dose was increased (IFX $n=2$ ). Intensification strategies in patients receiving IFX and ADA were carried out according to the drug levels obtained, while for VDZ it was performed according to signs of clinical activity and intestinal inflammation markers, such as CF.

Conclusion and relevance Biological drugs represent an effective and safe option in patients with UC but in approximately half of the cases in the study period, treatment had to be intensified. Therefore, the introduction into clinical practice of monitoring serum levels of biological drug is essential for a correct intensification strategy.

\section{REFERENCES AND/OR ACKNOWLEDGEMENTS}

No conflict of interest.

\section{CPS-154 POTENTIALLY INAPPROPRIATE MEDICATION FOR ELDERLY HOSPITALISED PATIENTS IN A TRAUMA AND ORTHOPAEDIC SURGERY DEPARTMENT}

MM Alañon Pardo*, MT Gómez Lluch, MC Conde García, B Proy Vega, ML Moreno Perulero, JC Valenzuela Gámez. Hospital General La Mancha-Centro, Pharmacy, Alcázar De San Juan, Spain

\subsection{6/ejhpharm-2020-eahpconf.255}

Background and importance Medication is potentially inappropriate when the risk of adverse effects is greater than the clinical benefit, especially when safer and/or more effective treatments are available.

Aim and objectives To analyse potentially inappropriate prescriptions (PIPs) and potential prescribing omissions (PPOs) in elderly patients hospitalised for trauma and/or orthopaedic surgery.

Material and methods A prospective observational study (15 August 2018-15 February 2019) was conducted in the trauma/orthopaedic surgery department of a general hospital. Inclusion criteria were age $\geq 65$ years, $\geq 3$ chronic medications and interview with a pharmacist for reconciliation of home medication at hospital admission. Study variables were sex, age, number of comorbidities, number and type of chronic medications, place of residence (home, or residential/health centre $(\mathrm{R} / \mathrm{HC})$ ) and reason for admission and its type (elective/urgent). Medications were categorised using the anatomical therapeutic chemical classification system. STOPP-START criteria were used to detect PIPs and PPOs. Binary logistic regression analysis was conducted to identify factors related to PIPs and POPs.

Results The study included 114 patients $(6.4 \%$ women, mean age $79.8 \pm 7.9$ years, $3.2 \pm 2.2$ comorbidities/patient, $7.9 \pm 3.6$ medications/patient; $6.1 \%$ in $\mathrm{R} / \mathrm{HC})$. The main reason for admission was hip fracture (45.6\%); $57.9 \%$ of admissions were urgent and due to falls. Among the 898 chronic medications evaluated, $15.8 \%$ were a PIP or PPO. The most frequently implicated anatomic groups were: A-alimentary tract/ metabolism (24.9\%), C-cardiovascular (24.2\%) and $\mathrm{N}$-nervous system (24.1\%). We detected 131 PIPs in 72 patients (63.2\%), including: STOPP-A1, medication without indication (18.3\%), mainly (75\%) proton pump inhibitors (PPIs); STOPP-A2, treatment longer than recommended (17.6\%); STOPP-A3, duplication (9.2\%); STOPP-K1, benzodiazepine in falls (7.6\%); and STOPP-D5, benzodiazepines $>4$ weeks (6.1\%), among others. We detected 15 PPOs in 13 patients $(11.4 \%)$, including: START-D1, gastroprotection with PPI (33.3\%); START-E3, calcium/vitamin D in osteoporosis (26.7\%); START-H2, laxative with opioid (20.0\%); and START-E5, vitamin D in elderly after fall (6.7\%), among others. The number of chronic medications per patient was the sole factor associated with PIPs and/or PPOs $(\mathrm{OR}=1.49$ (95\% CI 1.17-1.89), $\mathrm{p}=0.001$ ).

Conclusion and relevance PIPs were highly prevalent among elderly trauma patients; they were more frequent than PPOs and mainly attributable to polymedication. The medications most frequently associated with PIPs were PPIs and benzodiazepines, which can increase the risk of falls and hip fractures.

\section{REFERENCES AND/OR ACKNOWLEDGEMENTS}

1. Delgado-Silveira. Rev Esp Geriatr Gerontol 2015.

No conflict of interest. 